\title{
Current State and Future Perspectives in QSAR Models to Predict Blood Brain Barrier penetration in Central Nervous System Drug R\&D
}

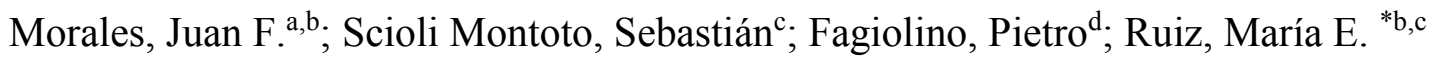 \\ ${ }^{a}$ Medicinal Chemistry, Department of Biological Sciences, Faculty of Exact Sciences, National University of La Plata (UNLP), \\ La Plata (B1900AJI), Buenos Aires, Argentina \\ ${ }^{b}$ Argentinean National Council for Scientific and Technical Research (CONICET) - CCT La Plata (B1900AJI), Buenos Aires, \\ Argentina
}

${ }^{c}$ Quality Control of Drugs, Department of Biological Sciences, Faculty of Exact Sciences, National University of La Plata (UNLP), La Plata (B1900AJI), Buenos Aires, Argentina

${ }^{d}$ Biopharmaceutics \& Therapeutics, Department of Pharmaceutical Sciences, Faculty of Chemistry, and Bioavailability and Bioequivalence Center for Medicine Evaluation, Universidad de la República, Montevideo, Uruguay

\begin{abstract}
The blood brain barrier (BBB) is a physical and biochemical barrier that restricts the entry of certain drugs to the Central Nervous System (CNS), while allowing the passage of others. The ability to predict the permeability of a given molecule through the BBB is a key aspect in CNS drug discovery and development, since neurotherapeutic agents with molecular targets in the CNS should be able to cross the BBB, whereas peripherally acting agents should not, to minimize the risk of CNS adverse effects. In this review we examine and discuss QSAR approaches and current availability of experimental data for the construction of BBB permeability predictive models, focusing on the modeling of the biorelevant parameter unbound partitioning coefficient (Kp,uu) . Emphasis is made on two possible strategies to overcome the current limitations of in silico models: considering the prediction of brain penetration as a multifactorial problem, and increasing experimental datasets through accurate and standardized experimental techniques.
\end{abstract}

Keywords: Blood-Brain Barrier, Brain Penetration, Central Nervous System, Microdialysis, Passive Difussion, Pharmacokinetic, Protein Binding, QSAR Models, Unbound Drug Fraction.

\section{INTRODUCTION}

In the field of Central Nervous System (CNS) drug R\&D, where crossing the blood brain barrier (BBB) is a key step for biodistribution, in silico models have been extensively used for the prediction of a drug's brain penetration ability, on the basis of different independent variables or descriptors. From the 1980 work by Levin et al, where quantitative relationships of rat brain capillary permeability coefficient with the octanol/water partition coefficient and molecular weight of 27 compounds were presented [1], many other models have been proposed and reviewed [2-6].

However, in recent years a significant change of paradigm has been evidenced, changing the BBB transport modeling approach from less to more biorelevant parameters, mainly due to the identification of unbound, pharmacologically active drug as the most relevant molecular entity $[7,8]$.

\footnotetext{
*Address correspondence to this author at Quality Control of Medications, Department of Biological Sciences, Faculty of Exact Sciences, National University of La Plata (UNLP), La Plata (B1900AJI), Buenos Aires, Argentina, Tel/Fax: +54-221-423-5333 ext. 43, E-mails: eruiz@biol.unlp.edu.ar, eruiz.unlp@gmail.com
}

The potential of the Quantitative Structure-Activity Relationship (QSAR) approach seems to be enormous: to predict not only the biological activity, but also the pharmacokinetic profile of a given compound (including its ability to cross de BBB), by expressing the desired property of a group of compounds as a function of several molecular descriptors, in order to generate a model that is then used to predict the same property for other compounds not included in the training set [9]. However, achieving good prediction accuracy with computational methods for estimating brain transport in early drug discovery still remains a challenging task.

The prediction errors due to poor application of statistical methods and recommended guidelines are two of the main reasons for the lack of prediction ability $[10,11]$. Back in 2009, Dearden et al. listed and discussed the most common of these problems, which can be roughly classified into failure of the data, failure of the descriptors used, failures in the generation, validation and/or interpretation of the model and failures in the statistical methods applied [12]. Furthermore, underlying any QSAR model is the notion that similar chemical structures mean similar biological activities 
or properties. The most common type of QSARs misuse is extrapolation beyond their domain. A QSAR is defined and limited by the nature and quality of the data used for its development, and it is applicable only to compounds that belong to the chemical classes included in the training set [13].

Hence, the current challenge is to expand QSARs applications while minimizing its limitations, which can only be done by developing better models, based in high-quality experimental data sets and more biorelevant descriptors and modeling parameters. For that reason this review begins discussing the current knowledge on the $\mathrm{BBB}$ and the models reported (and the parameters used) for the prediction of brain penetration based on the unbound concentration of the drug, as well as about the experimental methods currently available to determine that concentration, and some of the possible ways to optimize BBB penetration predictions. In particular, we believe that there is no single way to address the issue, and that future improvements will come from multifactorial approaches that take into account all of the different aspects of the problem and the complexity imposed by the BBB, as well as the multiplicity of interrelated processes that simultaneously occur when trying to deliver a drug to the CNS.

\section{BLOOD-BRAIN BARRIER PENETRATION}

Many drugs interact with plasma or tissue proteins, or other macromolecules such as melanin and DNA, to form a drug-macromolecule complex [14]. The formation of a drug protein complex (drug-protein binding) may be an irreversible or a reversible process, the latter being the most common one, with different binding strength for each drug. Reversible drug-protein binding is of major interest in pharmacokinetics (PK): the protein-bound drug is a large complex that cannot easily cross cell membranes and therefore has a restricted distribution. Moreover, the proteinbound drug is usually pharmacologically inactive. In contrast, the free or unbound fraction of the drug is able to distribute from the vascular space into tissues where it can interact with the therapeutic target to induce its pharmacodynamics response [15].

The aforementioned is usually called the "free-drug hypothesis", which in other words states that only the free, unbound fraction of a drug present at its site of action is responsible for the pharmacologic response and, since it is the only fraction able to cross cell membranes, free drug concentration is in equilibrium across those membranes (assuming only passive transport) at steady state [16]. The $\mathrm{BBB}$, possibly the most selective and tightly controlled of these barriers, is a clear example of this situation: formed by the endothelial cells of brain microvessels, the BBB is a dynamic multicellular structure that separates the CNS from the systemic circulation $[17,18]$.

Between the cells that form the BBB there are very tight lateral connections (tight junctions) that limit paracellular permeability of drugs and other compounds, which are thus forced to cross the BBB through the transcellular path (see Figure 1) [19]. This is the way of entry of most of the current therapeutic drugs used for the treatment of CNS diseases, usually small lipophilic molecules capable of passively crossing plasmatic membranes, like benzodiazepines or barbiturates, among many others. Two other mechanisms are available to pass through the BBB: the active transport route, mediated by influx and/or efflux transporters, and the endocytic route (receptor and/or adsorption mediated, only way of accessing for large molecules, nanoparticles and high molecular weight complexes) [20,21]. Efflux transporters of the ATP binding cassette family ( $\mathrm{ABC}$ transporters) deserve a special mention since they are responsible for extruding drugs from the brain, which in turn reduces drugs' CNS bioavailability [22-24]. Pglycoprotein (Pgp), Multidrug Resistant Protein (MRP) and Breast Cancer Resistant Protein (BCRP) are the three most studied members of this family, and its ability to move their substrates out of cells has been proved to be a major obstacle in CNS drug delivery [25-27].

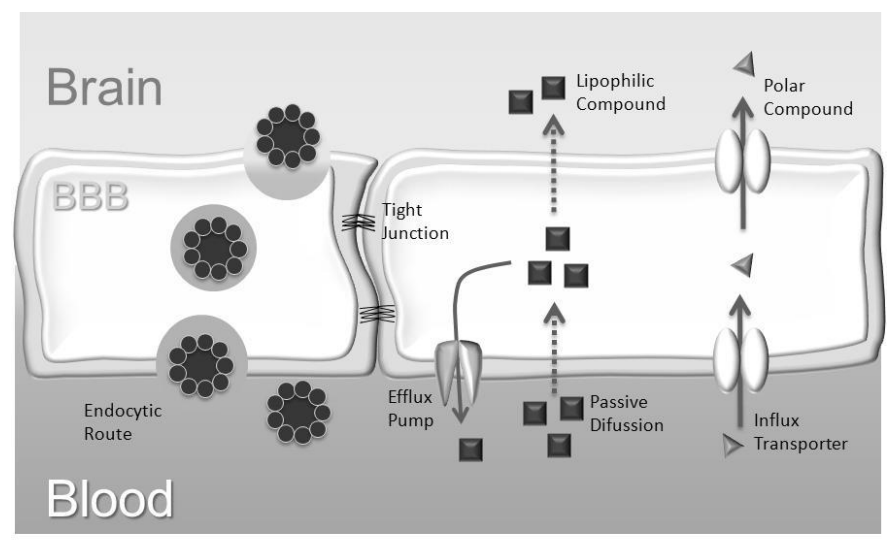

Figure 1. Transcellular transport routes across the BBB. In the scheme, three molecules (squares) enter the endothelial cell by passive diffusion through the membrane and once there, while two of them reach the CNS, the other one is captured and extruded by an efflux pump. The carrier-mediated influx and the endocytic pathway are also displayed.

Being the main transport route across the $\mathrm{BBB}$, the transcellular passive diffusion pathway is the most studied one, and the applicability of the free drug hypothesis has already been proved for a great variety of drugs with different targets and belonging to different therapeutic areas [28].

Keeping that in mind, it follows that the free drug fraction is a much more informative modeling parameter to describe the distribution equilibrium established across the BBB. Despite that plasma drug concentrations are generally reported as total concentrations (most therapeutic effective drug levels found in the literature refer to the total concentration), it is now widely accepted that the free levels lead to more informative (and accurate) PK/PD relations and QSAR models [28-32].

It is worth to highlight, however, that if the passive diffusion is low, or the active transport (both for efflux or influx of the drug to the tissue) is significant, an equilibrium with equal free levels at both sides of the BBB will not be reached. In other words, a new equilibrium with dissimilar 
free concentrations between plasma and CNS will be attained. Other exception to the applicability of the hypothesis are those drugs that irreversibly bind to its target, as well as the drugs with multiple receptors and/or mechanisms of action [16].

Figure 2 shows a schematic representation of the different fractions (bound/unbound) of a drug across the BBB. The scheme reflects that, despite both fractions exist on both sides of the membrane, only the free drug is able to diffuse and thus reaches the equilibrium at steady state.

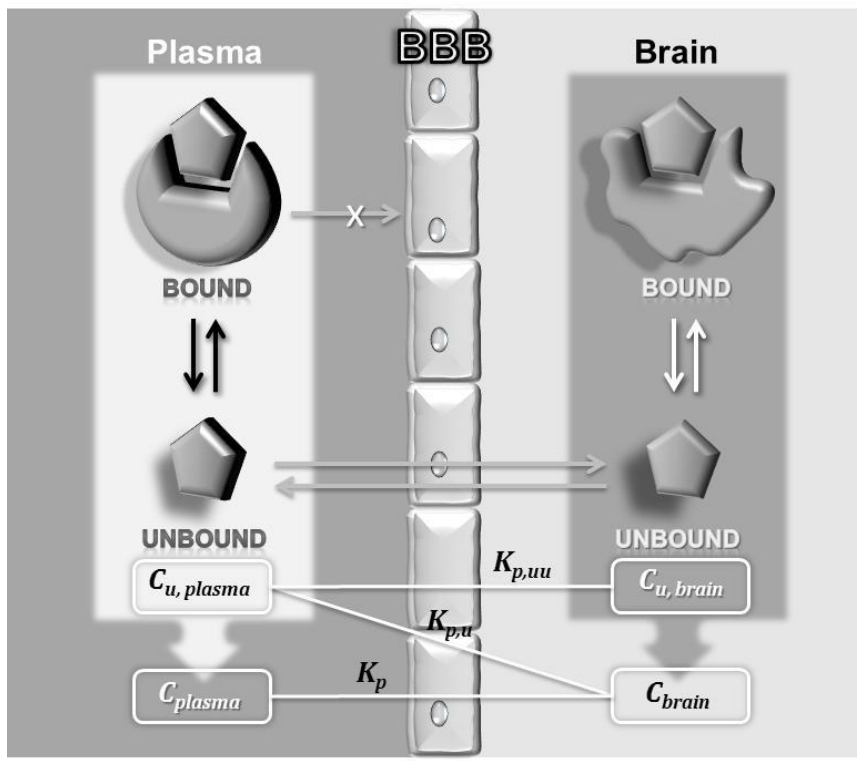

Figure 2. Schematic representation of the different equilibriums that a drug may experience at both sides of the BBB. The different plasma and brain drug concentrations and the corresponding concentration ratios are showed.

\subsection{Parameters that describe brain-to-plasma distribution}

Table 1 presents a summary of the main parameters that have been proposed to describe the distribution of drugs between brain and plasma $[9,33,34]$

As stated before, not all the proposed parameters are equally suitable to optimize lead drugs and/or to guide the $\mathrm{R} \& \mathrm{D}$ of new drug candidates. Among the most informative are unbound drug concentration in brain $\left(C_{u, \text { brain }}\right)$, unbound brain-to-unbound plasma ratio $\left(K_{p, u u}\right)$, and brain-to-unbound plasma ratio $\left(K_{p, u}\right)$. The parameter $K_{p, u}$ only eliminates the effect of binding in plasma and is defined as the ratio of the total brain $\left(C_{\text {brain }}\right)$ to unbound plasma $\left(C_{u, p l a s m a}\right)$ concentrations of the drug. Based on the assumption of passive transport, $K_{p, u}$ gives a measure of the percentage of protein binding in brain tissue [9]

Parameters like $k_{i n}$ or $P S$ focus on the rate of influx of drugs from plasma to brain, but give no information about the distribution process that follows. In a very interesting work, Summerfield et al. studied the relationship between $k_{\text {in }}$ and the parameters $K_{p}$ and $K_{p, u u}$, finding a poor correlation in both cases, that the authors attributed to the fact that both $K_{p}$ and $K_{p, u u}$ are concentration distribution ratios and thus do not provide information about the rate of entry of drugs into the brain [35].

Other parameters like total brain-to-plasma ratio (or partition ratio, $\left.K_{p}\right)$ and its logarithmic expression $(\log B B)$, as well as the unbound fraction in plasma or in brain $\left(f_{\text {u,plasma }}\right.$ and $f_{u, b r a i n}$, respectively) provide little information to understand or predict the in vivo efficacy of drugs into the CNS [29]. Despite that, $\log B B$ is clearly the most popular quantitative parameter and has been widely used by the pharmaceutical industry to determine the efficiency of drug distribution in the CNS $[8,9,35]$. By using models based on $\log B B$ it is only possible to establish the total amount of drug in the brain once the distribution balance has been reached, but not to determine the corresponding free drug concentration, responsible for the pharmacological effect according to the free drug hypothesis discussed above $[35,36]$.

A clear example of the limited utility of $\log B B$ to describe a drug's pharmacological effects on the CNS can be found in a 2009 work by Watson et al., where the authors clearly demonstrated that in vivo occupancy of $\mathrm{D}_{2}$ receptors in rats was better correlated with $C_{u \text {, brain }}$ (normalized by the in vitro affinity of the drug for the $\mathrm{D}_{2}$ receptor). Furthermore, given the penetration in terms of $K_{p}$, Risperidone was the drug with the lowest CNS entry, but that was dismissed because the drug possessed the highest free concentration associated with its high $\mathrm{D}_{2}$ potency [28].

First introduced by Gupta et al. [37] the concept of $K_{p, u u}$ is essential to assess if the distribution balance between blood and brain have been established. As showed first by Gupta and then by Bostrom et al. [38], this parameter provides information about the mechanism of transport across the BBB, and it is sensible to a compound's affinity for the efflux/influx transporters found in the epithelial cells. Compounds with good passive permeability and that are not substrates of efflux transporters (e.g. Pgp, BCRP) typically present $K_{p, u u}$ values close to 1 . In the same manner, $K_{p, u u}$ values less than 1 could indicate that a compound is substrate for an efflux transporter and/or it has limited brain penetration due to low passive permeability across the BBB, while $K_{p, u u}$ values greater than 1 suggests active uptake by influx transporters $[8,29,35]$.

As stated before, possibly due to the easier experimental determination of $\log B B$ (which in turns leads to a greater availability of experimental data), in silico models using $L o g$ $B B$ are abundant in scientific literature, even in recent years [39-44], when the disadvantages and limitations of this parameter have been recognized [45-47]. Loryan et al. have recently studied the relationship between $K_{p}$ and $K_{p, u u}$, finding no correlation between the parameters and hence concluding that different conclusions will arise from each of them [48].

Therefore, the next sections of this review will focus on the most biorelevant parameter $K_{p, u u}$. Both the revision and the analysis of currently available $\log B B$ (and $\log P S$ ) computational models can be found elsewhere (see for example $[5,9,41,47,49-51]$ and references therein). 


\section{HOW TO MEASURE DRUG TRANSPORT ACROSS THE BBB}

All QSAR models rely on a set of experimental data, which size and quality determine the model prediction accuracy: widely scattered experimental data, for example, will incorporate their variability to the model [51,52], in the same manner that lower quality data is associated with a poorer statistical fit and usually lead to inaccurate predictions $[13,53]$

Computational models to predict CNS bioavailability of drugs are not an exception, and the widespread use of $\log$ $B B$ highlights the lack of methods for measuring the free brain fraction of drugs in an efficient manner [54]. In other words, while abundant descriptions can be found for the experimental determination of $\log P S$ and $\log B B$ [9,35,51,55], determining a compound's $K_{p, u u}$ is a much harder task, that involves the experimental quantification of its free plasma and brain concentrations, both at the steady state.

The gold standard techniques to measure the free drug fraction in plasma $\left(f_{u \text {,plasma }}\right)$ are equilibrium dialysis and ultrafiltration; the free plasma drug concentration $\left(C_{u, p l a s m a}\right)$ is then calculated by knowing the total plasma concentration $\left(C_{\text {plasma, }}\right.$ usually determined by chromatographic or immunologic methods). They are however time-consuming experimental techniques that require large sample volumes, among other drawbacks [56]. Therefore, other approaches to assess $C_{u \text {,plasma }}$ have been proposed using several other experimental techniques (spectroscopy, calorimetry, HPLC, capillary electrophoresis and surface plasmon resonance based biosensors [57,58]), corrective algorithms like the Sheiner-Tozer equation $[59,60]$, and even the use of saliva, as a natural plasma ultrafiltrate, for the direct quantification of the free drug concentration $[61,62]$.
On the other hand, the direct quantification of the free concentration in brain $\left(C_{u, \text { brain }}\right)$ is possible only through in vivo intracerebral microdialysis (MD) [63,64], which involves the implantation of a probe into the brain by stereotaxic surgery. This invasive method is usually performed in rodents, and most commonly in rats. The probe is a tubular dialysis membrane with inlet and outlet tubes for perfusion and sample collection, which is perfused with artificial brain extracellular fluid [65], to assure that the diffusion of compounds is due only to the concentration gradient across the semipermeable membrane. Quantification of the drug is then performed in the sampled dialysate. An important aspect of the technique is that it requires an additional step for the determination of the recovery from the probe, because usually the concentration equilibrium at both sides of the membrane is not reached, and thus the concentration in the dialysate is lower than the true extracellular concentration in the brain [47,66-68].

Despite being the gold standard, MD in rodents has several disadvantages that prevent it to become a high throughput technique: it is an expensive and time-consuming methodology that can only be performed by highly trained personnel. These drawbacks are most probably the cause of the insufficient $K_{p, u u}$ data available to build QSAR models, and also the reason why other experimental techniques to determine $C_{u \text {, brain }}$ have been proposed [69].

Here, it is important to underline that even though the use of CSF concentrations has been proposed as an estimation of free brain concentration, it was demonstrated that both concentrations do not always correlate well $[37,72,77,79]$. On the other hand, the use of animal models to determine free brain concentrations is justified since human data are of limited use for QSAR modeling since intracerebral MD may be performed only in human patients suffering from a CNS or BBB affecting disease, and under very strict conditions [70].

Table 1. Main parameters proposed to describe a drug's brain-to-plasma distribution

\begin{tabular}{|c|c|c|}
\hline Parameter & Expression & Meaning \\
\hline$C_{\text {plasma }}$ and $C_{\text {brain }}$ & & $\begin{array}{l}\text { Total concentrations in plasma and brain, which are the sum of the unbound and } \\
\text { bound drug concentrations in each fluid. }\end{array}$ \\
\hline$C_{u, \text { plasma }}$ and $C_{u, \text { brain }}$ & $\begin{array}{l}C_{u, \text { plasma }}=f_{u, \text { plasma }} * C_{\text {plasma }} \\
C_{u, \text { brain }}=f_{u, \text { brain }}{ }^{*} C_{\text {brain }}\end{array}$ & $\begin{array}{l}\text { Free, diffusible and therapeutically active concentration of the drug in plasma } \\
\text { and brain, respectively. }\end{array}$ \\
\hline$f_{u, \text { plasma }}$ and $f_{u, \text { brain }}$ & & Fraction of unbound drug in plasma and brain, respectively. \\
\hline$K_{p}$ & $K_{p}=C_{\text {brain }} / C_{\text {plasma }}$ & The overall steady-state brain/plasma distribution ratio \\
\hline $\log B B$ & $\log B B=\log \left(K_{p}\right)$ & Logarithmic expression of the total brain to plasma ratio \\
\hline$K_{p, u}$ & $K_{p, u}=C_{\text {brain }} / C_{u, \text { plasma }}$ & Total brain to unbound plasma steady-state concentrations ratio \\
\hline
\end{tabular}


The homogenate technique first implemented by Kalvass and Maurer in 2002 involves placing a small sample of brain homogenate (brain tissue diluted with buffer and homogenized with a sonic probe) spiked with the drug under study into a 96-well equilibrium dialysis apparatus, and dialyzing versus fresh buffer $[73,74]$. Knowing the overall steady-state brain concentration $\left(C_{\text {brain }}\right)$ and determining the free fraction $\left(f_{u, \text { brain }}\right)$ by the previously described dialysis experiment, $C_{u \text {, brain }}$ can be obtained. Therefore, it is a highthroughput technique suitable for CNS drug discovery [75], but with one major weakness: the homogenization process may modify the tissue's binding properties, exposing additional drug binding sites and thus potentially leading to an underestimation of the free fraction $[69,76]$.

Another method to estimate the free drug concentration in brain is the slice method [76,77], which consists in the incubation at $37{ }^{\circ} \mathrm{C}$ of brain slices (of around $400 \mu \mathrm{m}$ in width) of rat or mouse in plasma or buffer spiked with a given amount of the test compound/s. To determine the

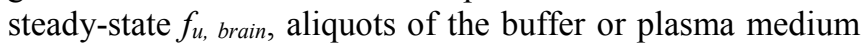
are withdrawn at designated time points, and the test compound is quantified by a suitable technique. As the homogenate technique, the slice method has also been developed as a high throughput one, with the additional advantage of allowing the maintenance of cell structure [78].

In a very interesting work, Fridén et al. compared the performance of the three methods mentioned above in a set of 15 compounds. Despite no significant differences were found for the in vitro and in vivo techniques, the slice method had a slightly better performance than the homogenate method, most probably due to the maintenance of the tissue viability, and the preservation of the distribution processes of the brain [77].

In conclusion, the MD technique should be the first choice to measure the free drug concentration in brain. However, if a screening of several drug candidates is intended, the slice method could be a suitable option to obtain good $f_{u}$, brain estimations within optimized times. Finally, the use of the homogenate method is not dismissed, taking into account the limitations of the method, which measures non-specific binding of drugs [79].

\section{QSAR MODELS TO PREDICT BBB PENETRATION}

As said before, QSAR models for the prediction of brain penetration were usually based on the $K_{p}$ parameter, and we could only find four works that use $K_{p, u u}$ as modeling parameter $[45,46,54,80]$. Table 2 presents a summary of these four QSAR models reviewed in this section.

A computational model for the logarithmic expression of the unbound partition coefficient, $\log K_{p, u u}$, was first published by Fridén et al. [54], who obtained two models by partial least squares (PLS): one with 16 molecular 2D descriptors and the other with the HBA (number of hydrogen bond acceptors) descriptor only, with a cross-validated coefficient of correlation [81] $\left(Q^{2}\right)$ of 0.452 and 0.426 , respectively. The training set consisted on 41 marketed drugs with a 300 -fold range of $K_{p, u u}$ values obtained by the slice method in steady state (equivalent to a 10000 -fold range of $K_{p}$ values). Besides the internal validation of the models, the authors performed an external validation with a test set of 54 and $91 K_{p, u u}$ data obtained by the microdialysis and homogenate techniques, respectively. The result of this external validation, measured by the root mean squared error (RMSE) value for the 16-molecular descriptor model and the HBA model were 3.99 and 4.19, respectively.

Despite that the results showed that only the $40-45 \%$ of the total variation could be explained by the models, it is interesting to observe that the most significant molecular descriptors were those related with the hydrogen bonding properties (polar surface area -PSA- and HBA), and the model with only the HBA descriptor showed that it is necessary to remove two hydrogen bonds to achieve a 2-fold increase in the $K_{p, u u}$ value.

Paradoxically, a $\log B B$ model derived for the same training set and based on descriptors related to the lipophilicity, basicity and hydrogen bonding properties of the compounds showed better relative predictivity $\left(Q^{2}=0.693\right)$, but the authors advice against using it for drug design, since it would result in the selection of unnecessarily lipophilic or basic compounds without improved pharmacodynamics [54].

The moderate prediction power of Fridén's models could be due to the reduced size of the training set and the few descriptors calculated that could have prevented the achievement of a good SAR, and the low cases/independent variables ratio of the model with 16 molecular descriptors (around 2.5 cases of the training set per independent variable used). Additionally, the authors were not clear about the curation of the chemical structures prior to the descriptors' calculation. Overall, these factors (along with the large variability of data sources included in the test set) could have diminished the prediction accuracy of the models. Nevertheless, the work of Fridén et al. led to a new understanding of the factors affecting the passage of drugs through the BBB.

The next report on a $K_{p, u u}$ QSAR model is a 2011 work by Chen et al [80]. Using Random Forest (RF) and Support Vector Machine (SVM) algorithms, the authors derived direct and indirect regression models that were then combined in consensus $K_{p, u u}$ models. While direct models were based on available $K_{p, u u}$ data, indirect models were a combination of primary (or individual) models of total brainto-plasma ratio $\left(K_{p}\right)$, unbound volume of distribution in the brain $\left(V_{u, \text { brain }}\right)$ and unbound fraction of drug in plasma $\left(f_{u, p l a s m a}\right)$, according to the following equation:

$$
K_{p, u u}=\frac{K_{p}}{V_{u, \text { brain }} \times f_{u, \text { brain }}}
$$


Table 2. Summary of the QSAR models developed using $K_{p, u u}$ as modeling parameter. When the authors derived more than one model for the same data set, the $R^{2}$ and RMSE values of the best model are presented. PLS: partial least squares, SVM: support vector machine, RF: random forest, $R^{2}$ : coefficient of correlation, $Q^{2}$ : cross-validated coefficient of correlation, RMSE: root mean squared error.

\begin{tabular}{|c|c|c|c|c|c|c|}
\hline \multirow{2}{*}{ Reference } & \multirow{2}{*}{$\begin{array}{l}\text { Statistical } \\
\text { Analysis }\end{array}$} & \multirow{2}{*}{ Descriptors } & \multirow{2}{*}{ Data Set } & \multirow{2}{*}{$N$} & \multicolumn{2}{|c|}{ Test Set } \\
\hline & & & & & $R^{2}$ & RMSE \\
\hline Friden et al. [54] & PLS & $162 \mathrm{D}$ descriptors & training set & 41 & $0.452\left(Q^{2}\right)$ & 3.99 \\
\hline \multirow{2}{*}{ Chen et al. [80] } & \multirow{2}{*}{ SVM, RF } & \multirow{2}{*}{$1962 \mathrm{D}$ and 3D descriptors } & training set & 173 & \multirow{2}{*}{0.58} & \multirow{2}{*}{0.46} \\
\hline & & & test set & 73 & & \\
\hline Loryan et al. [45] & PLS & $1881 \mathrm{D}, 2 \mathrm{D}$ and $3 \mathrm{D}$ descriptors & test set & 11 & 0.82 & 0.31 \\
\hline \multirow{2}{*}{ Varadharajan et al. [46] } & \multirow{2}{*}{ SVM, RF } & \multirow{2}{*}{$\begin{array}{l}196 \text { 2D and 3D descriptors; } \\
\text { signature descriptors }\end{array}$} & training set & 242 & \multirow{2}{*}{0.65} & \multirow{2}{*}{0.45} \\
\hline & & & test set & 104 & & \\
\hline
\end{tabular}

Figure 3 shows the model building scheme and the number of compounds in each data set. The indirect models were necessary to take advantage of the larger amount of available $K_{p}, V_{u \text {,brain }}$ and $f_{u \text {,plasma }}$ data compared to the $K_{p, u u}$ data. The consensus models were obtained by averaging the predictions of two or more individual $K_{p, u u}$ models. The direct, indirect and consensus models were validated with a test set of 73 compounds with $K_{p, u u}$ values obtained by the slice method at stationary state [80].

The authors calculated a total of 196 2D and 3D descriptors, and found that those that were relevant for $K_{p, u u}$ were not for $K_{p}$ : while $K_{p, u u}$ was highly correlated with Kappa2 descriptor (an estimation of molecular linearity) and no correlation was observed with ACD $\log \mathrm{P}(\log \mathrm{P})$. These observations support the previous ones by Fridén et al. [54] about the independency of $K_{p, u u}$ from molecular lipophilicity (as a measure of a compound's ability to interact with a biologic membrane) and its relationship with other parameters (hydrogen bonding capacity, molecular linearity) that account for its interaction with membrane transporters. The best model obtained was one of the consensus models, with a correlation coefficient $\left(R^{2}\right)$ of 0.58 , and the lowest RMSE (0.46) for the test set.

The authors also studied the classification ability of the models, by using a $K_{p, u u}$ threshold value of 0.1 : compounds with $K_{p, u u} \geq 0.1$ were regarded as having good brain bioavailability, and poor brain exposure was considered if $K_{p, u u}<0.1$. According to this classification criterion, the same consensus model showed the best performance, with $85 \%$ and $89 \%$ of good classifications (\%GC) in the training and test set, respectively. It is important to remark, however, that all the compounds in the test set presented good brain bioavailability.

Among the reasons why the models of Chen et al. [80] showed a better performance than those obtained by Fridén et al. [54] we can cite the inclusion of more compounds in the training set, the calculation of more molecular descriptors and the implementation of consensus models. Moreover, the application of a classification criteria allowed the authors to evaluate the information with very good predictive power even though the arbitrary choice of the threshold value. On the other hand, the great variability of data sources in the training set of the indirect models may have been a source of prediction errors.

A recent work by Loryan et al. [45] derived $K_{p, u и}$ based QSAR models using training and test sets comprising 39 and 11 compounds, respectively, with $K_{p, u u}$ values obtained by the slice method, but instead of working at the stationarystate, $K_{p}$ values used for calculation were based on single dose studies (oral or subcutaneous) [48]. A total of 188 1D, $2 \mathrm{D}$ and $3 \mathrm{D}$ molecular descriptors were calculated, and the models were obtained by PLS regression. The best model obtained presented the following values: $R^{2}=0.76$ and $Q^{2}=$ 0.72 for the training set, and $R^{2}=0.82$ and $\mathrm{RMSE}=0.31$ for the test set. The model comprised two molecular descriptors: vsurf Cw8 and TPSA, both related to the compounds' polarity and hydrogen bonding ability, and the correlation was inverse: an increase in the descriptors' value cause a decrease of the $K_{p, u u}$ value.

The developed model showed poor performance when the compounds from Friden's data set [54] that fell within the applicability domain (30 of 41) were used as external test set, indicating that TPSA and vsurf Cw8 descriptors cannot adequately explain the variance in the $K_{p, u u}$ for this 30 compounds. Conversely, a good correlation was found when other two descriptors (vsurf_W1 and a_don, also related with the polarity and the ability to form hydrogen bonds) were used, suggesting that local models for a given data set could be an option to improve the prediction results or that alternatively, broader and more diverse datasets are still required.

As in the previously reviewed studies, the hydrogen bonding capacity also proved to be an important factor affecting the transport across the BBB. However, the model of Loryan et al. is the first that also evidences the influence of polarity on the value of $K_{p, u u}$. 


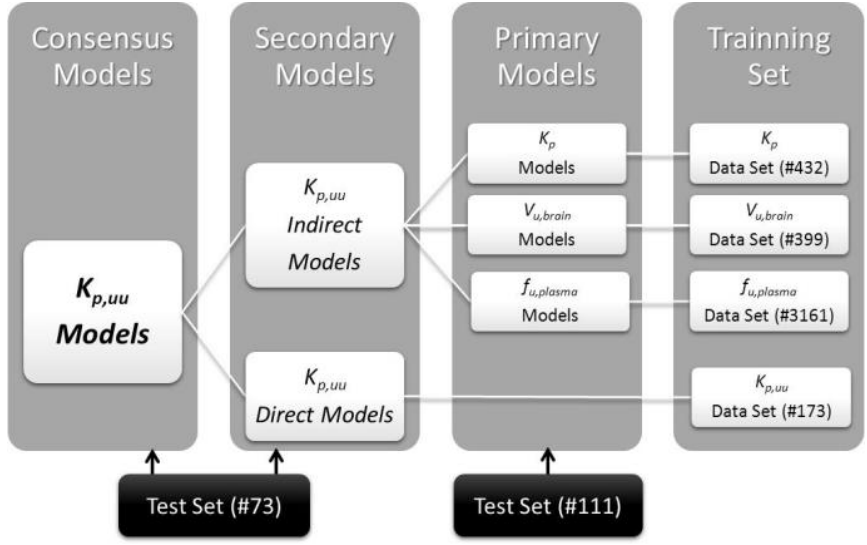

Figure 3. Diagram of the methodology applied by Chen et al. to build the consensus, direct and indirect models [80]. The number of compounds in each data set is indicated in parentheses.

While it may seem that this model has better performance than the previous two, the model of Chen has still the best performance in an external test set. As stated earlier, the data set used by Loryan et al. consisted in approximated $K_{p, u u}$ values, calculated with $K_{p}$ values obtained after a single dose administration. Although it has been proposed that this is a valid approach to obtain good approximations to the true $K_{p, u u}$ value [48,82], only when the stationary state is reached all the distribution equilibriums across de BBB are established.

The last article reviewed is a 2015 study published by Varadharajan et al. [46] that presents an expansion (and additional validation) of the 2011 work by Chen et al, by the addition of new data and new descriptors. In general, the methodology was similar and two data sets of $K_{p, u u}$ obtained by the slice method at stationary-state were included: in addition to the original 247 compounds (data set 1), a second set of 99 new $K_{p, \text { uu }}$ data were included (data set 2), as well as a third set consisting on the combination of the previous two (i.e. 346 compounds, data set 3). Atom-based descriptors were used to describe the extended valence of the atoms in the molecules (signature descriptors). Besides the 2-class classification system (CS) presented in 2011, the authors proposed a 3-class CS into low, medium and high brain bioavailability according to the threshold $K_{p, u u}$ values of 0.3 and 0.05 .

The validation of the original model (built with data set 1 ) with the data set 2 yielded $R^{2}$, RMSE and $\% \mathrm{GC}$ values of $0.53 \%, 0.58$ and $76 \%$, respectively, which were slightly lower than those reported in 2011. In addition, the \%GC using the 3-class CS were $92 \%, 40 \%$ and $83 \%$ for high, medium and low classes respectively. The worst value observed for the medium class seems to indicate that the model could distinguish between high or low brain exposures, but not intermediate exposure compounds.

The authors also developed six new models based on data set 3, which were then combined to build consensus models. As in 2011, two consensus models displayed the best performance with the highest average $R^{2}$ and RMSE values
( 0.65 and 0.45 , respectively). For the 2-class CS the $\% \mathrm{GC}$ was near $84 \%$ for the best consensus models, a value similar to the one obtained for the high and low classes of the 3class CS (around 80\%). However, as in the previous case, a poor classification precision was found for the moderate class ( $46 \%$ of GC).

From these results, it can be seen that the new models (with the expanded data set) performed slightly better than the ones built with data set 1 . Despite that, the 3 -class CS did not represent any improvement, possibly due to the fact that every classification model possesses poor performance near the threshold values [83], which in this case were two instead of one. A possible and simple solution would be to define different threshold values according to the intended use of the model. For example, to search for BBB-crossing compounds, a conservative criterion could be used (e.g. $K_{p, u u}$ $>0.6$ ) to assure high prediction specificity.

As a structural interpretation of the models, the authors presented the analysis of each atom contribution to the predicted value for 8 compounds (atom-summarized gradient contributions), an approach that could prove useful to guide molecular design in a drug discovery program.

Although the results presented above are promising in terms of prediction of the passage through the BBB, all the reviewed studies have in common the lack of sufficient $K_{p, u u}$ data. The available data sets are not only limited but also often imprecise, noisy or even contradictory and generated from different experimental protocols. Moreover, another factor that could jeopardize the models predictivity is the fact that the unbound fraction is not a single value to be assigned to any concentration for a particular drug, but instead different unbound fractions are observed for many drugs depending on their concentration [16].

Taken altogether, these considerations, rather than discourage the use of QSAR modeling for CNS drug R\&D, encourage us to keep looking for new and better alternatives to improve the prediction precision and accuracy of the models.

\section{FUTURE PERSPECTIVES}

Analyzing the trend in new drugs R\&D through the years, it can be acknowledged that, after a golden era in the late 90 s, the pharmaceutical industry experienced a decline in research productivity, where despite that the R\&D investment increased substantially, the lack of a corresponding increase in the output was alarming, with the lowest number of new drug applications approved between 2005-2010 [84-86]. Moreover, when analyzed by therapeutic areas, CNS is unfortunately among the less productive areas [87]. The most worrying aspect of this unfavorable statistics is that their overall effect is to discourage the investment in therapeutic areas where the chances for medical or scientific breakthroughs are lower [85]. In other words, the research for new molecular entities for the treatment of CNS disorders is in danger of presenting a downward trend in the coming years $[88,89]$. 
In contrast, the incidence and socio-economical cost of CNS diseases are increasing at an upsetting rate and, according to a 2014 report of the World Health Organization, the burden of mental disorders continues to grow with significant impacts on health and major social, human rights and economic consequences all over the world [90].

When trying to explain the main reasons for the failure in drug discovery and development, the arguments found usually belong to one of the next categories: drug metabolism and pharmacokinetic (DMPK) issues, toxicological issues, efficacy issues, clinical safety and/or commercial issues [84]. However, in the case of CNS disorders DMPK issues have always been regarded as the most troublesome due to the presence of the BBB as a major obstacle for the drugs to reach its active site inside the CNS.

Despite that transport across the BBB depends mainly on the nature of the drug, its free plasma concentration and its ability to be captured by efflux transporters, there are many other factors that may influence the concentration gradient established through the BBB, like binding to brain tissue, enzymatic metabolism and/or the presence of different entry routes for the same compound, among others. Brain metabolism, for example, may affect the free concentration of a drug in the CNS, since metabolizing enzymes present at the BBB and/or brain tissue can influence the bioavailability of drugs in the brain [91]. Whereas all these processes can occur simultaneously and each of them can influence the rate and extent of the others, it is very important to take all these inter-relationships into account when predicting brain exposure [8].

An interesting example of how various PK and PD factors can be interrelated is found when analyzing the effect of the already mentioned efflux transporters (ABC transporters). While it has been shown that the increased efflux transport in Caco-2 cells may decrease the parameter $K_{p, u u}$ [92], another study found that among 24 compounds that exhibited low $K_{p, u u}$ values $\left(K_{p, u u}<0.5\right)$, only 10 were identified as Pgp substrates, while several others of low and even very low $K_{p, u u}$ values $\left(K_{p, u u}<0.1\right)$ were not [48]. The antipsychotic drug Paliperidone belongs to the latter category, highlighting the importance of considering the PD potency when analyzing the likelihood of success of CNS drug candidates, as well as extending of these studies to other transporters overexpressed at the BBB.

There are still many challenges in order to improve the prediction accuracy of QSAR models applied to pharmacokinetic properties. For example, classic models (like the ones reviewed here) are not able to properly describe the PK profile of drugs that modify their own biodistribution during chronic or prolonged use, like the anticonvulsant phenytoin, which induces its own extrusion from the CNS by inducing the expression of efflux transporters [93]. While this effect might be seen in an animal model of chronic treatment with the drug, other effects may not, like the influence of circadian rhythms, physiopathology and/or cardiovascular physiology of the patient on the distribution and disposition of the drug. Aging, as another example, leads to an increased blood flow delivery to the extra-splanchnic-renal region with a decreased cardiac output, but brain and myocardium have their cardiac output fraction increased in the elderly [94]. As a consequence, changes in plasma drug concentration may not proportionally translate in changes at the action site outside the vessels [95]. In other words, elderly and young patients could have the same free plasma concentration of a drug but different therapeutic effect, as has been reported for Diazepam and Digoxin [96,97].

Multifactorial approaches have already proven to be very promising strategies for the optimization of CNS drugs R\&D $[8,29,48,70]$. The workflow proposed by $\mathrm{Di}$ et al. is a clear example of that: with parallel and sequential stages, it allows optimizing the screening for new CNS drug candidates by simultaneously seeking for the reduction of Pgp/BCRP efflux transport [98], the minimization of metabolic and systemic clearance reactions, and the increase of both permeability and solubility of the drug (Figure 4) [29].

In a study published in 2013, de Lange proposed a "Mastermind Approach", as a way to learn about the contributions of individual processes in drug PK-PD relationships through advanced mathematical modeling. The author proposed a model based on data of plasma PK, brain distribution and CNS effects of a drug, in which timedependency should also be included, as well as information on the unbound drug fraction [70]. Alternatively, Ball et al. developed a population physiologically based pharmacokinetic model (population PBPK model) of the CNS based on preclinical brain microdialysis data [99]. This multifactorial approach was able to accurately predict brain free drug concentrations in rats, and it is the first PBPK model based on free drug fractions. Along with the aforementioned approaches, we think that this could be the beginning of a new trend in predicting CNS bioavailability during early drug discovery.

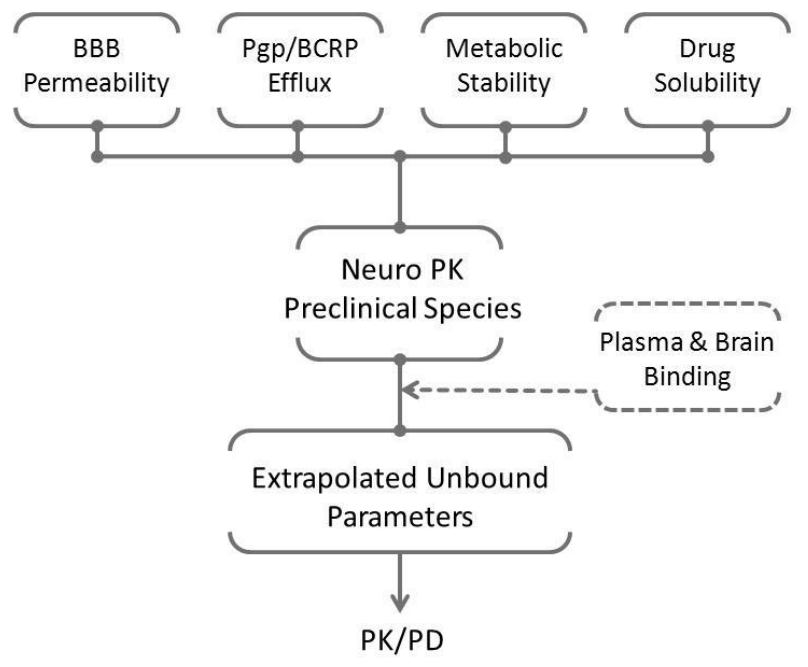

Figure 4. Screening workflow for CNS new drug candidates. Adapted with permission from $D i$ et al. Demystifying Brain Penetration in Central Nervous System Drug Discovery. J Med Chem 56(1): 2-12. Copyright 2012 (C) American Chemical Society 


\section{CONCLUSION}

In order to potentiate the discovery and development of new drug candidates for treating CNS diseases, efforts have been focused on these physiologic barrier and, as we previously discussed, QSAR modeling became one of the main and most promising methodologies for the discovery of new drugs and/or new indications for known drugs (drug repurposing) $[100,101]$.

However, over the previous sections it has also been revealed that computational modeling of a single parameter by itself may not be sufficient to achieve a definitive solution to the CNS drug discovery problem, not only due to the limitations of the in silico methodology and the currently scarce available data sets, but also because of the multifactorial nature of the addressed issue.

It seems that the research and the need for new CNS drugs are two sides of the same coin with opposite trends, where the challenge is to find the best approaches, or combination of them, to offer new therapeutic opportunities for treating these kinds of diseases. For sure, a better understanding of the variability sources in the $\mathrm{PK} / \mathrm{PD}$ modeling of CNS drugs will help improve the development of new drug candidates and therapies [70].

\section{CONFLICT OF INTEREST}

The author(s) confirm that this article content has no conflicts of interest.

\section{ACKNOWLEDGEMENTS}

We thank the American Chemical Society for the permission to reproduce Figure 4. The authors would like to thanks UNLP and CONICET. J. F. Morales is a fellowship holder from the National Council of Scientific and Technical Research (CONICET). M. E. Ruiz is a member of the Scientific Research Career at CONICET.

J. F. Morales, S. Scioli Montoto and M. E. Ruiz thank Incentivos UNLP.

\section{REFERENCES}

[1] Levin, V.A. Relationship of octanol/water partition coefficient and molecular weight to rat brain capillary permeability. J. Med. Chem., 1980; 23(6), 682-4.

[2] Feng, M.R. Assessment of blood-brain barrier penetration: in silico, in vitro and in vivo. Curr. Drug Metab., 2002; 3(6), 647-57.

[3] Pardridge, W.M. $\log (\mathrm{BB})$, PS products and in silico models of drug brain penetration. Drug Discov. Today, 2004; 9(9), 392-3.

[4] Kumar, R.; Sharma, A.; Tiwari, R.K. Can we predict blood brain barrier permeability of ligands using computational approaches? Interdiscip. Sci., 2013; 5(2), 95-101.

[5] Mehdipour, A.R.; Hamidi, M. Brain drug targeting: a computational approach for overcoming blood-brain barrier. Drug Discov. Today, 2009; 14(21-22), 1030-6.

[6] Suenderhauf, C.; Hammann, F.; Huwyler, J. Computational prediction of blood-brain barrier permeability using decision tree induction. Molecules, 2012; 17(9), 10429-45.

[7] Hammarlund-Udenaes, M.; Fridén, M.; Syvänen, S.; Gupta, A. On the rate and extent of drug delivery to the brain. Pharm. Res., 2008; 25(8), 1737-50.

[8] De Lange, E.C.M.; Hammarlund-Udenaes, M. Translational aspects of blood-brain barrier transport and central nervous system effects of drugs: from discovery to patients. Clin. Pharmacol. Ther., 2015; 97(4), 380-94.

[9] Lanevskij, K.; Japertas, P.; Didziapetris, R. Improving the prediction of drug disposition in the brain. Expert Opin. Drug Metab. Toxicol., 2013; 9(4), 473-86.

[10] Tropsha, A. Best practices for QSAR model development, validation, and exploitation. Mol. Inform., 2010; 29(6-7), 476-88.

[11] Tropsha, A.; Golbraikh, A. Predictive QSAR modeling workflow, model applicability domains, and virtual screening. Curr. Pharm. Des., 2007; 13(34), 3494-504.

[12] Dearden, J.C.; Cronin, M.T.D.; Kaiser, K.L.E. How not to develop a quantitative structure-activity or structure-property relationship (QSAR/QSPR). SAR QSAR Environ. Res., 2009; 20(3-4), 241-66.

[13] Walker, J.D.; Jaworska, J.; Comber, M.H.I.; Schultz, T.W.; Dearden, J.C. Guidelines for developing and using Quantitative Structure-Activity Relationships. Environ. Toxicol. Chem., 2003; 22(8), 1653.

[14] Shargel, L.; Yu, A.B.C. Applied biopharmaceutics and pharmacokinetics. 3rd ed. Prentice-Hall International: London, 1993.

[15] Roberts, J.A.; Pea, F.; Lipman, J. The clinical relevance of plasma protein binding changes. Clin. Pharmacokinet., 2013; 52(1), 1-8.

[16] Smith, D.A.; Di, L.; Kerns, E.H. The effect of plasma protein binding on in vivo efficacy: misconceptions in drug discovery. Nat. Rev. Drug Discov., 2010; 9(12), 929-39.

[17] Abbott, N.J.; Rönnbäck, L.; Hansson, E. Astrocyte-endothelial interactions at the blood-brain barrier. Nat. Rev. Neurosci., 2006; $7(1), 41-53$.

[18] Keaney, J.; Campbell, M. The Dynamic Blood-Brain Barrier. FEBS $J ., 2015$; doi: $10.1111 /$ febs. 13412 .

[19] Hawkins, B.T.; Davis, T.P. The blood-brain barrier/neurovascular unit in health and disease. Pharmacol. Rev., 2005; 57(2), 173-85.

[20] Begley, D.J.; Brightman, M.W. Structural and functional aspects of the blood-brain barrier. Prog. Drug Res., 2003; 61, 39-78.

[21] Pardridge, W.M. Blood-brain barrier drug targeting: the future of brain drug development. Mol. Interv., 2003; 3(2), 90-105, 51.

[22] Vasiliou, V.; Vasiliou, K.; Nebert, D.W. Human ATP-binding cassette (ABC) transporter family. Hum. Genomics, 2009; 3(3), 281-90.

[23] Chen, Y.; Liu, L. Modern methods for delivery of drugs across the blood-brain barrier. Adv. Drug Deliv. Rev., 2012; 64(7), 640-65.

[24] Vlieghe, P.; Khrestchatisky, M. Medicinal chemistry based approaches and nanotechnology-based systems to improve CNS drug targeting and delivery. Med. Res. Rev., 2013; 33(3), 457-516.

[25] Ejendal, K.F.; Hrycyna, C.A. Multidrug resistance and cancer: the role of the human ABC transporter ABCG2. Curr. Protein Pept. Sci., 2002; 3(5), 503-11.

[26] Marquez, B.; Van Bambeke, F. ABC multidrug transporters: target for modulation of drug pharmacokinetics and drug-drug interactions. Curr. Drug Targets, 2011; 12(5), 600-20.

[27] Talevi, A.; Bruno-Blanch, L.E. In: The Blood-Brain Barrier: New Research; Montenegro P.A. \& Juárez S.M. Eds.; Nova Publishers: New York, 2012; pp. 117-44.

[28] Watson, J.; Wright, S.; Lucas, A.; Clarke, K.L.; Viggers, J.; Cheetham, S.; Jeffrey, P.; Porter, R.; Read, K.D. Receptor occupancy and brain free fraction. Drug Metab. Dispos., 2009; 37(4), 753-60.

[29] Di, L.; Rong, H.; Feng, B. Demystifying Brain Penetration in Central Nervous System Drug Discovery. J. Med. Chem., 2012; $56(1), 2-12$.

[30] Hammarlund-Udenaes, M. Active-site concentrations of chemicals - Are they a better predictor of effect than plasma/organ/tissue concentrations? Basic \& Clinical Pharmacology \& Toxicology, 2010; 106(3), 215-20.

[31] Liu, Z.; Sall, A.; Yang, D. MicroRNA: An emerging therapeutic target and intervention tool. Int J Mol Sci, 2008; 9(6), 978-99.

[32] Kalvass, J.C.; Olson, E.R.; Cassidy, M.P.; Selley, D.E.; Pollack, G.M. Pharmacokinetics and pharmacodynamics of seven opioids in P-glycoprotein-competent mice: assessment of unbound brain EC50,u and correlation of in vitro, preclinical, and clinical data. $J$. Pharmacol. Exp. Ther., 2007; 323(1), 346-55.

[33] Jeffrey, P.; Summerfield, S.G. Challenges for blood-brain barrier (BBB) screening. Xenobiotica., 2008; 37(10-11), 1135-51. 
[34] Hammarlund-Udenaes, M.; Fridén, M.; Syvänen, S.; Gupta, A. On the rate and extent of drug delivery to the brain. Pharm. Res., 2008; 25(8), 1737-50.

[35] Summerfield, S.G.; Dong, K.C. In vitro, in vivo and in silico models of drug distribution into the brain. J. Pharmacokinet. Pharmacodyn., 2013; 40(3), 301-14.

[36] Reichel, A. Addressing central nervous system (CNS) penetration in drug discovery: Basics and implications of the evolving new concept. Chem. Biodivers., 2009; 6(11), 2030-49.

[37] Gupta, A.; Chatelain, P.; Massingham, R.; Jonsson, E.N.; Hammarlund-Udenaes, M. Brain distribution of cetirizine enantiomers: Comparison of three different tissue-to-plasma partition coefficients: Kp, Kp,u, and Kp,uu. Drug Metab. Dispos., 2006; 34(2), 318-23.

[38] Boström, E.; Simonsson, U.S.H.; Hammarlund-Udenaes, M. In vivo blood-brain barrier transport of oxycodone in the rat: indications for active influx and implications for pharmacokinetics/pharmacodynamics. Drug Metab. Dispos., 2006; 34(9), 1624-31.

[39] Gupta, S.; Basant, N.; Singh, K.P. Qualitative and quantitative structure-activity relationship modelling for predicting blood-brain barrier permeability of structurally diverse chemicals. SAR QSAR Environ. Res., 2015; 26(2), 95-124.

[40] Golani, M.; Golani, I. On a Simulation Approach to Drug Blood Brain Barrier Permeation: Neural Net Ensemble Based. IAENG Int J. Comput. Sci., 2015; 42(1), 31-40.

[41] Bujak, R.; Struck-Lewicka, W.; Kaliszan, M.; Kaliszan, R.; Markuszewski, M.J. Blood-brain barrier permeability mechanisms in view of quantitative structure-activity relationships (QSAR). $J$. Pharm. Biomed. Anal., 2015; 108, 29-37.

[42] Wang, W.; Kim, M.T.; Sedykh, A.; Zhu, H. Developing Enhanced Blood-Brain Barrier Permeability Models: Integrating External Bio-Assay Data in QSAR Modeling. Pharm. Res., 2015; 32(9), 3055-65.

[43] Golmohammadi, H.; Dashtbozorgi, Z.; Acree, W.E. Quantitative structure-activity relationship prediction of blood-to-brain partitioning behavior using support vector machine. Eur. J. Pharm. Sci., 2012; 47(2), 421-9.

[44] Brito-Sánchez, Y.; Marrero-Ponce, Y.; Barigye, S.J.; YaberGoenaga, I.; Morell Pérez, C.; Le-Thi-Thu, H.; Cherkasov, A. Towards Better BBB Passage Prediction Using an Extensive and Curated Data Set. Mol. Inform., 2015; 34(5), 308-30.

[45] Loryan, I.; Sinha, V.; Mackie, C.; Van Peer, A.; Drinkenburg, W.H.; Vermeulen, A.; Heald, D.; Hammarlund-Udenaes, M.; Wassvik, C.M. Molecular properties determining unbound intracellular and extracellular brain exposure of CNS drug candidates. Mol. Pharm., 2015; 12(2), 520-32.

[46] Varadharajan, S.; Winiwarter, S.; Carlsson, L.; Engkvist, O.; Anantha, A.; Kogej, T.; Fridén, M.; Stalring, J.; Chen, H. Exploring in silico prediction of the unbound brain-to-plasma drug concentration ratio: model validation, renewal, and interpretation. J. Pharm. Sci., 2015; 104(3), 1197-206.

[47] Di, L.; Kerns, E.H. Blood-Brain Barrier in Drug Discovery. John Wiley \& Sons, Inc: Hoboken, NJ, 2015.

[48] Loryan, I.; Sinha, V.; Mackie, C.; Van Peer, A.; Drinkenburg, W.; Vermeulen, A.; Morrison, D.; Monshouwer, M.; Heald, D.; Hammarlund-Udenaes, M. Mechanistic understanding of brain drug disposition to optimize the selection of potential neurotherapeutics in drug discovery. Pharm. Res., 2014; 31(8), 2203-19.

[49] Raevsky, O.A.; Solodova, S.L.; Lagunin, A.A.; Poroikov, V. V. Computer modeling of blood brain barrier permeability for physiologically active compounds. Biochem. Suppl. Ser. B Biomed. Chem., 2013; 7(2), 95-107.

[50] Subramanian, G.; Kitchen, D.B. Computational models to predict blood-brain barrier permeation and CNS activity. J. Comput. Aided. Mol. Des., 2003; 17(10), 643-64.

[51] Mensch, J.; Oyarzabal, J.; Mackie, C.; Augustijns, P. In vivo, in vitro and in silico methods for small molecule transfer across the BBB. J. Pharm. Sci., 2009; 98(12), 4429-68.

[52] Fourches, D.; Muratov, E.; Tropsha, A. Trust, but verify: on the importance of chemical structure curation in cheminformatics and QSAR modeling research. J. Chem. Inf. Model., 2010; 50(7), 1189204.
Cronin, M.T.; Schultz, T.W. Validation of Vibrio fisheri acute toxicity data: mechanism of action-based QSARs for non-polar narcotics and polar narcotic phenols. Sci. Total Environ., 1997; 204(1), 75-88.

[54] Fridén, M.; Winiwarter, S.; Jerndal, G.; Bengtsson, O.; Wan, H.; Bredberg, U.; Hammarlund-Udenaes, M.; Antonsson, M. Structurebrain exposure relationships in rat and human using a novel data set of unbound drug concentrations in brain interstitial and cerebrospinal fluids. J. Med. Chem., 2009; 52(20), 6233-43.

[55] Abbott, N.J. Prediction of blood-brain barrier permeation in drug discovery from in vivo, in vitro and in silico models. Drug Discov. Today. Technol., 2004; 1(4), 407-16.

[56] Vuignier, K.; Veuthey, J.-L.; Carrupt, P.-A.; Schappler, J. Global analytical strategy to measure drug-plasma protein interactions: from high-throughput to in-depth analysis. Drug Discov. Today, 2013; 18(21-22), 1030-4.

[57] Vuignier, K.; Schappler, J.; Veuthey, J.-L.; Carrupt, P.-A.; Martel, S. Drug-protein binding: a critical review of analytical tools. Anal. Bioanal. Chem., 2010; 398(1), 53-66.

[58] Yang, G.X.; Li, X.; Snyder, M. Investigating metabolite-protein interactions: an overview of available techniques. Methods, 2012; 57(4), 459-66.

[59] Hong, J.M.; Choi, Y.C.; Kim, W.J. Differences between the measured and calculated free serum phenytoin concentrations in epileptic patients. Yonsei Med. J., 2009; 50(4), 517-20.

[60] Bolt, J.; Gorman, S.K. Precision, bias, and clinical utility of the Sheiner-Tozer equation to guide phenytoin dosing in critically ill adults. J. Clin. Pharmacol., 2013; 53(4), 451-5.

[61] Ibarra, M.; Vázquez, M.; Fagiolino, P.; Mutilva, F.; Canale, A. Total, Unbound Plasma and Salivary Phenytoin Levels in Critically Ill Patients. J Epilepsy Clin Neurophysiol, 2010; 16(2), 69-73.

[62] Fagiolino, P.; Vazquez, M.; Maldonado, C.; Ruiz, M.E.; Volonte, M.G.; Orozco-Suarez, S.; Lazarowski, A. Usefulness Of Salivary Drug Monitoring For Detecting Efflux Transporter Overexpression. Curr. Pharm. Des., 2013; 19(38), 6701-8.

[63] Spreafico, M.; Jacobson, M.P. In silico prediction of brain exposure: drug free fraction, unbound brain to plasma concentration ratio and equilibrium half-life. Curr. Top. Med. Chem., 2013; 13(7), 813-20.

[64] Ungerstedt, U. Microdialysis--principles and applications for studies in animals and man. J. Intern. Med., 1991; 230(4), 365-73.

[65] Zapata, A.; Chefer, V.I.; Shippenberg, T.S. Microdialysis in rodents. Curr. Protoc. Neurosci., 2009; 47(7.2), 1-29.

[66] Müller, M. Microdialysis in Drug Development. Springer: New York, 2013.

[67] Di Giovanni, G.; Di Matteo, V. Microdialysis Techniques in Neuroscience. Humana Press: Totowa, NJ, 2013.

[68] Chefer, V.I.; Thompson, A.C.; Zapata, A.; Shippenberg, T.S. Overview of brain microdialysis. Curr. Protoc. Neurosci., 2009; 47(7.1), 1-28.

[69] Read, K.D.; Braggio, S. Assessing brain free fraction in early drug discovery. Expert Opin. Drug Metab. Toxicol., 2010; 6(3), 337-44.

[70] De Lange, E.C. The mastermind approach to CNS drug therapy: translational prediction of human brain distribution, target site kinetics, and therapeutic effects. Fluids Barriers CNS, 2013; 10(1), $1-12$.

[71] Bickel, U. How to measure drug transport across the blood-brain barrier. NeuroRx, 2005; 2(1), 15-26.

[72] Smith, Q.R. A review of blood-brain barrier transport techniques. Methods Mol. Med., 2003; 89, 193-208.

[73] Kalvass, J.C.; Maurer, T.S. Influence of nonspecific brain and plasma binding on CNS exposure: implications for rational drug discovery. Biopharm. Drug Dispos., 2002; 23(8), 327-38.

[74] Mano, Y.; Higuchi, S.; Kamimura, H. Investigation of the high partition of YM992, a novel antidepressant, in rat brain - in vitro and in vivo evidence for the high binding in brain and the high permeability at the BBB. Biopharm. Drug Dispos., 2002; 23(9), 351-60.

[75] Wan, H.; Rehngren, M.; Giordanetto, F.; Bergström, F.; Tunek, A. High-throughput screening of drug-brain tissue binding and in silico prediction for assessment of central nervous system drug delivery. J. Med. Chem., 2007; 50(19), 4606-15. 
[76] Becker, S.; Liu, X. Evaluation of the utility of brain slice methods to study brain penetration. Drug Metab. Dispos., 2006; 34(5), 85561.

[77] Fridén, M.; Gupta, A.; Antonsson, M.; Bredberg, U.; HammarlundUdenaes, $M$. In vitro methods for estimating unbound drug concentrations in the brain interstitial and intracellular fluids. Drug Metab. Dispos., 2007; 35(9), 1711-9.

[78] Fridén, M.; Ducrozet, F.; Middleton, B.; Antonsson, M.; Bredberg, U.; Hammarlund-Udenaes, M. Development of a high-throughput brain slice method for studying drug distribution in the central nervous system. Drug Metab. Dispos., 2009; 37(6), 1226-33.

[79] Fridén, M.; Bergström, F.; Wan, H.; Rehngren, M.; Ahlin, G.; Hammarlund-Udenaes, M.; Bredberg, U. Measurement of unbound drug exposure in brain: modeling of $\mathrm{pH}$ partitioning explains diverging results between the brain slice and brain homogenate methods. Drug Metab. Dispos., 2011; 39(3), 353-62.

[80] Chen, H.; Winiwarter, S.; Fridén, M.; Antonsson, M.; Engkvist, O. In silico prediction of unbound brain-to-plasma concentration ratio using machine learning algorithms. J. Mol. Graph. Model., 2011; 29(8), 985-95.

[81] Wold, S. Validation of QSAR's. Quant. Struct. Relationships, 1991; 10(3), 191-3.

[82] Doran, A.C.; Osgood, S.M.; Mancuso, J.Y.; Shaffer, C.L. An evaluation of using rat-derived single-dose neuropharmacokinetic parameters to project accurately large animal unbound brain drug concentrations. Drug Metab. Dispos., 2012; 40(11), 2162-73.

[83] Gramatica, P. On the development and validation of QSAR models. Computational toxicology, 2013; 930, 499-526.

[84] Khanna, I. Drug discovery in pharmaceutical industry: productivity challenges and trends. Drug Discov. Today, 2012; 17(19-20), 1088-102.

[85] Pammolli, F.; Magazzini, L.; Riccaboni, M. The productivity crisis in pharmaceutical R\&D. Nat. Rev. Drug Discov., 2011; 10(6), 42838

[86] Scannell, J.W.; Blanckley, A.; Boldon, H.; Warrington, B. Diagnosing the decline in pharmaceutical R\&D efficiency. Nat. Rev. Drug Discov., 2012; 11(3), 191-200.

[87] Smietana, K.; Ekstrom, L.; Jeffery, B.; Møller, M. Improving R\&D productivity. Nat. Rev. Drug Discov., 2015; 14(7), 455-6.

[88] Hurko, O.; Ryan, J.L. Translational research in central nervous system drug discovery. NeuroRx, 2005; 2(4), 671-82.

[89] Pangalos, M.N.; Schechter, L.E.; Hurko, O. Drug development for CNS disorders: strategies for balancing risk and reducing attrition. Nat. Rev. Drug Discov., 2007; 6(7), 521-32.
[90] WHO. Mental disorders - Fact sheet $\mathrm{N}^{\circ} 396$, October 2014 http://www.who.int/mediacentre/factsheets/fs396/en/ (Accesed August 8, 2015)

[91] Hammarlund-Udenaes, M.; de Lange, E.C.M.; Thorne, R.G. Drug Delivery to the Brain. Springer: New York, 2014.

[92] Plowright, A.T.; Nilsson, K.; Antonsson, M.; Amin, K.; Broddefalk, J.; Jensen, J.; Lehmann, A.; Jin, S.; St-Onge, S.; Tomaszewski, M.J.; Tremblay, M.; Walpole, C.; Wei, Z.; Yang, H.; Ulander, J. Discovery of agonists of cannabinoid receptor 1 with restricted central nervous system penetration aimed for treatment of gastroesophageal reflux disease. J. Med. Chem., 2013; 56(1), 220 40 .

[93] Alvariza, S.; Ibarra, M.; Vázquez, M.; Fagiolino, P. Different Phenytoin Oral Administration Regimens Could Modify Its Chronic Exposure and Its Saliva/Plasma Concentration Ratio. $J$ Med Pharm Innov., 2014; $1(6 \mathrm{~S}), 35-43$.

[94] Fagiolino, P.; Eiraldi, R.; Vázquez, M. The influence of cardiovascular physiology on dose/pharmacokinetic and pharmacokinetic/pharmacodynamic relationships. Clin. Pharmacokinet., 2006; 45(5), 433-48.

[95] Fagiolino, P. The influence of cardiac output distribution on the tissue/plasma drug concentration ratio. Eur J Drug Metab Pharmacokinet, 2002; 27(2), 79-81.

[96] Hämmerlein, A.; Derendorf, H.; Lowenthal, D.T. Pharmacokinetic and pharmacodynamic changes in the elderly. Clinical implications. Clin. Pharmacokinet., 1998; 35(1), 49-64.

[97] Miura, T.; Kojima, R.; Sugiura, Y.; Mizutani, M.; Takatsu, F.; Suzuki, Y. Effect of aging on the incidence of digoxin toxicity. Ann. Pharmacother., 2000; 34(4), 427-32.

[98] Gantner, M.E.; Di Ianni, M.E.; Ruiz, M.E.; Talevi, A.; BrunoBlanch, L.E. Development of conformation independent computational models for the early recognition of breast cancer resistance protein substrates. Biomed Res. Int., 2013; 2013, 863592.

[99] Ball, K.; Bouzom, F.; Scherrmann, J.-M.; Walther, B.; Declèves, X. Comparing translational population-PBPK modelling of brain microdialysis with bottom-up prediction of brain-to-plasma distribution in rat and human. Biopharm. Drug Dispos., 2014; 35(8), 485-99.

[100] Cherkasov, A.; Muratov, E.N.; Fourches, D.; Varnek, A.; Baskin, I.I.; Cronin, M.; et al. QSAR modeling: where have you been? Where are you going to? J. Med. Chem., 2014; 57(12), 4977-5010.

[101] Sliwoski, G.; Kothiwale, S.; Meiler, J.; Lowe, E.W. Computational methods in drug discovery. Pharmacol. Rev., 2014; 66(1), 334-95. 